\title{
An Efficient Joint Timing and Frequency Offset Estimation for OFDM Systems
}

\author{
Yang Yang*, Qing Huang ${ }^{\dagger}$ and Zaichen Zhang ${ }^{\dagger}$ \\ ${ }^{*}$ School of Information Science and Engineering \\ Southeast University, 210096, Nanjing, China \\ Email: yangyang.1388@gmail.com \\ ${ }^{\dagger}$ National Mobile Communications Research Laboratory \\ Southeast University, 210096, Nanjing, China
}

\begin{abstract}
This paper presents a new joint frame synchronization and frequency offset estimation algorithm for orthogonal frequency division multiplexing (OFDM) systems as a modification to Zhang's method [1]. By designing a new training preamble weighted by $\mathrm{PN}$ sequence, the timing estimator is improved (at least 3dB with low SNR). By estimating time offset first, the computational load is greatly reduced with no loss in frequency offset estimation accuracy. The performance of the proposed method is evaluated by computer simulations in terms of timing error rate (TER) together with computational complexity.
\end{abstract}

\section{INTRODUCTION}

Orthogonal frequency division multiplexing (OFDM) has been widely used in communication systems such as digital audio broadcasting (DAB), digital video broadcasting (DVB) and asymmetric digital subscriber line (ADSL) modems together with wireless local area network (LAN) because of its robustness to inter symbol interference (ISI) and high efficiency in making use of bandwidth resources. However, it is very sensitive to synchronization errors caused by multipath delay and frequency shift as well as oscillator instability. To overcome this disadvantage, several approaches, data-aided or non-data-aided, have been proposed to perform timing synchronization and frequency offset estimation either jointly or individually.

Data-aided schemes are more suitable for applications requiring fast and reliable synchronization. A popular algorithm to perform timing synchronization and frequency offset estimation jointly is proposed by Schmidl [2]. This algorithm has a timing metric, which is robust to frequency offset, used to judge the beginning of the training symbol. However, it has a plateau which introduces uncertainty when judging the starting point. Besides, two training symbols are required to estimate frequency offset, decreasing the overall system efficiency.

Kim's algorithm [3] uses only one symbol for both timing and frequency offset estimation. However, its frequency offset estimation is based on perfect symbol timing, an assumption which cannot be guaranteed due to the plateau.

To eliminate the plateau inherent in Schmidl and Kim algorithm, several modifications are made. Minn's [4] method

This work is supported by National 863 project 2007AA01Z2B1 and NSFC project 60802005 . is free from the plateau. Nevertheless, the performance deteriorates when the number of subcarrier is small and/or in multipath fading channel. An approach proposed by Park [5] yields an impulse shaped timing metric, but it suffers from side-peaks. [6] and [7] propose timing and frequency offset synchronization methods suitable for preambles with constant envelope.

Zhang's method [1] is able to get more accurate frequency offset estimation than that of Schmidl. This improvement, however, is at the expense of far heavier computational load. Furthermore, its timing estimator is not precise enough to ensure satisfactory operation of frequency offset estimator.

In this paper, a timing estimator with the training preamble weighted by PN sequence similar to [6] is adopted as a modification to Zhang's method. On one hand, it achieves better timing synchronization performance. On another hand, Zhang's algorithm is greatly simplified with no loss in frequency offset estimation accuracy.

This paper is organized as follows. Section 2 gives the system model. In Section 3, Zhang's algorithm is briefly described. Section 4 is about the proposed algorithm performing joint timing synchronization and frequency offset estimation. Section 5 gives simulation results and computational complexity analysis. Finally Section 6 concludes the whole paper.

\section{SySTEM MODEL}

The OFDM samples at the outputs of the IFFT are given by

$$
x_{i}=\frac{1}{\sqrt{N}} \sum_{k=1}^{N_{u}-1} X_{k} \cdot e^{j 2 \pi i k / N}
$$

where $X_{k}$ is the complex modulated symbol on the $k$-th subcarrier, $N_{u}$ and $N$ is the size of subcarriers and IFFT, respectively. An OFDM symbol is denoted as

$$
x=\left\{x_{N-G}, \ldots, x_{N-1}, x_{0}, x_{1}, \ldots, x_{N-1}\right\}
$$

The first $\mathrm{G}$ samples, as same as the last $\mathrm{G}$ ones of the training symbol, are added to make up of the cyclic prefix (CP).

At the receiver, the received samples are modeled as

$$
y(i)=\exp (j 2 \pi \varepsilon i / N+\varphi) \sum_{l=0}^{N_{L}-1} h_{l} x\left(i-\tau_{l}\right)+n(i)
$$


where $\tau_{l}$ is the unknown arrival time of a symbol, $\varepsilon$ is the carrier frequency offset normalized to subcarrier spacing, $\varphi$ is the initial phase, $n_{i}$ is the sample of zero mean complex Gaussian noise process with variance $\sigma_{i}^{2}$ and $N_{L}$ is the number of resolvable path.

Synchronization of an OFDM signal requires finding and compensating the symbol timing and carrier frequency offset.

\section{The Available Timing And Frequency Offset Estimation Method [1]}

Samples in the preamble satisfy two conditions:

$$
\begin{gathered}
\text { Condition } 1: x_{i}=x_{i+N / 2}, \quad i=0,1, \ldots, N / 2-1 \\
\text { Condition } 2: x_{i}=x_{N-i}, \quad i=1,2, \ldots, N / 2-1
\end{gathered}
$$

Such a sequence could be generated in the frequency domain by modulating only even numbered subcarriers and the modulating data are symmetric about the $N / 2$-th subcarrier, i.e.,

$$
X_{i}=X_{N-i}, \quad i=1,2, \ldots, N / 2
$$

In the receiver, the relationship between a transmitted sample and its received version is

$$
r_{i}=x_{i-\theta} \cdot e^{j 2 \pi \varepsilon i / N}+n_{i}
$$

where $\theta$ is the correct timing point.

Zhang's algorithm is divided into two stages: acquisition mode and tracking mode. In the former stage, timing and coarse frequency offsets are estimated simultaneously while in the latter stage, a fine adjustment algorithm is taken to estimate the remaining carrier frequency offset. 2:

In the acquisition mode, define a vector based on Condition

$$
\begin{aligned}
\Psi_{i} & =\left[\Psi_{i}(1), \Psi_{i}(2), \ldots, \Psi_{i}(N / 2-1)\right] \\
& =\left[\frac{\Gamma_{i}(N / 2-1)}{\left|\Gamma_{i}(N / 2-1)\right|}, \frac{\Gamma_{i}(N / 2-2)}{\left|\Gamma_{i}(N / 2-2)\right|}, \ldots, \frac{\Gamma_{i}(1)}{\left|\Gamma_{i}(1)\right|}\right]
\end{aligned}
$$

where $\Gamma_{i}(k)=r(i+N-k) \cdot r^{*}(i+k)$ and:

$$
\Phi_{i}(f)=\sum_{k=1}^{N / 2-1} \Psi_{i}(k) \cdot e^{-j 2 \pi f k}
$$

where $|f| \leq 1 / 2$.

At the correct timing point, (8) could be simplified as follows:

$$
\Psi_{\theta}=\left[e^{j 2 \pi \varepsilon \cdot 2 / N}, e^{j 2 \pi \varepsilon \cdot 4 / N}, \ldots, e^{j 2 \pi \varepsilon \cdot(N-2) / N}\right]
$$

From (10) it is known that $\Phi_{\theta}(f)$ gets its maximum value at $f=2 \varepsilon / N$, i.e.,

$$
\hat{f}=\arg \max \left\{\left|\Phi_{\theta}(f)\right|^{2}\right\}
$$

Accordingly, the carrier frequency offset is coarsely estimated from equation $\hat{\varepsilon}=N \hat{f} / 2$.

Note that $\left|\Phi_{i}(f)\right|^{2}$ gets its maximum value only when both $\hat{\theta}$ and $\hat{f}$ are estimated correctly. Hence, timing and coarse frequency offset estimation could be performed at the same time by searching the maximum value in (12) at the bottom of the page, where $(L+1)$ is the length of the time window, $\triangle f$ is the frequency resolution and $2 \cdot K \cdot \triangle f$ equals the coarse estimation range of the frequency window, which is up to half the total signal bandwidth.

Assume the largest element in (12) is $\left|\Phi_{j}(i \cdot \Delta f)\right|^{2}$, then $\hat{\theta}=j, \hat{\varepsilon}=(N \cdot i \cdot \Delta f) / 2$.

In the tracking mode, only the remaining frequency offset $\varepsilon_{R}$ is left:

$$
\varepsilon_{R}=\varepsilon-\hat{\varepsilon}
$$

For a given $N$ and $\varepsilon$, the value of $\varepsilon_{R}$ is determined by that of $\triangle f$. A fine adjustment is deployed to estimate $\varepsilon_{R}$. Based on Condition 1, a carrier frequency offset fine adjustment algorithm is derived as:

$$
\hat{\varepsilon_{1}}=\frac{\operatorname{angle}\left\{\sum_{k=0}^{N / 2-1} r(\theta+k+N / 2) r^{*}(\theta+k)\right\}}{\pi}
$$

which is just the Schmidl's algorithm.

Based on Condition 2, $\varepsilon_{R}$ could be estimated as:

$$
\hat{\varepsilon_{2}}=\frac{N \sum_{k=1}^{N / 2-1}|D(k)| \cdot(N-2 k) \cdot \operatorname{angle}\{D(k)\}}{2 \pi \sum_{k=1}^{N / 2-1}|D(k)| \cdot(N-2 k)^{2}}
$$

where $D(k)=r(\theta+N-k) \cdot r^{*}(\theta+k)$.

Estimation range of the carrier frequency offset fine adjustment algorithm is only $\pm N / 2(N-2)$ subcarrier spacing, imposing the following restriction on $\triangle f$ :

$$
|\triangle f|<1 / 2(N-2)
$$

When we consider both Condition 1 and Condition 2, a fine adjustment estimator with weighted factors can be derived as:

$$
\hat{\varepsilon}=\frac{\hat{\varepsilon_{1}}+\rho \cdot \hat{\varepsilon_{2}}}{1+\rho}
$$

where $\rho$ is the weighted factor and $0 \leq \rho \leq 1$. The larger $\rho$ is, the more accurate $\hat{\varepsilon}$ is. When $\rho$ is 0 , the frequency offset estimator is reduced to the Schmidl's algorithm.

$$
\Lambda_{2 K+1, L+1}=\left[\begin{array}{cccc}
\left|\Phi_{0}(K \cdot \triangle f)\right|^{2} & \left|\Phi_{1}(K \cdot \triangle f)\right|^{2} & \cdots & \left|\Phi_{L}(K \cdot \triangle f)\right|^{2} \\
\vdots & \vdots & \ddots & \vdots \\
\left|\Phi_{0}(\triangle f)\right|^{2} & \left|\Phi_{1}(\triangle f)\right|^{2} & \cdots & \left|\Phi_{L}(\triangle f)\right|^{2} \\
\left|\Phi_{0}(0)\right|^{2} & \left|\Phi_{1}(0)\right|^{2} & \cdots & \left|\Phi_{L}(0)\right|^{2} \\
\left|\Phi_{0}(-\triangle f)\right|^{2} & \left|\Phi_{1}(-\triangle f)\right|^{2} & \cdots & \left|\Phi_{L}(-\triangle f)\right|^{2} \\
\vdots & \vdots & \ddots & \vdots \\
\left|\Phi_{0}(-K \cdot \triangle f)\right|^{2} & \left|\Phi_{1}(-K \cdot \triangle f)\right|^{2} & \cdots & \left|\Phi_{L}(-K \cdot \triangle f)\right|^{2}
\end{array}\right]
$$




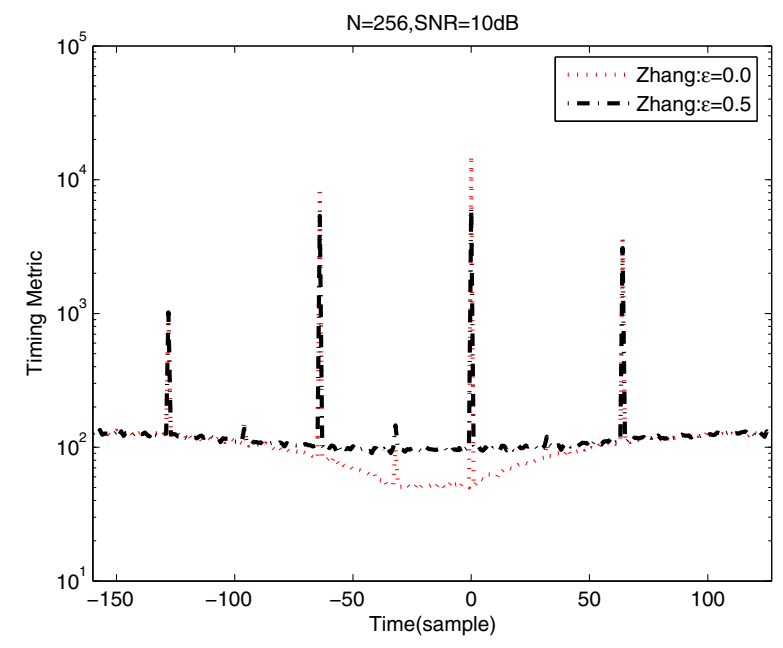

Fig. 1. Timing Metric of Zhang's Algorithm

\section{Proposed Synchronization And Frequency OfFSET Estimation Method}

Zhang's method performs the timing synchronization and coarse frequency offset estimation simultaneously. However, several factors make the algorithm unsatisfactory.

1) Zhang's timing estimator is greatly influenced by the remaining carrier frequency offset and the timing metric has three discernible side-peaks which could not be eliminated even though $\varepsilon_{R}$ is zero, as shown in Fig. 1. This may lead to wrong judgments.

2) The computational load to get the matrix in (12) is too heavy to make the algorithm feasible. The matrix is composed of $(2 K+1) \cdot(L+1)$ elements and none of the elements could be calculated iteratively. The number of elements becomes prohibitive when that of subcarriers is large, making the algorithm difficult to realize.

The above factors would prevent Zhang's algorithm from working correctly and effectively. In order to improve Zhang's timing estimator performance and simplify the algorithm, a modified method based on [1] and [6] is proposed.

In the proposed method, a single training symbol is used to estimate timing and frequency offset jointly. Firstly, the starting point of the proposed preamble is determined, so that the original complex matrix would be simplified into a one dimensional one. Then the coarse frequency offset in the acquisition mode and fine adjustment algorithm in the tracking mode would be proceeded.

The Schmidl timing estimator is robust to frequency offset. Nevertheless, there is a plateau in the timing metric which results in error when we try to determine the exact timing point of the symbol. This is because the values of the timing metric around the correct starting point are almost the same [5] [6]. To enlarge the difference between two adjacent values of the timing metric, a training preamble with PN sequence weighted factor is introduced.

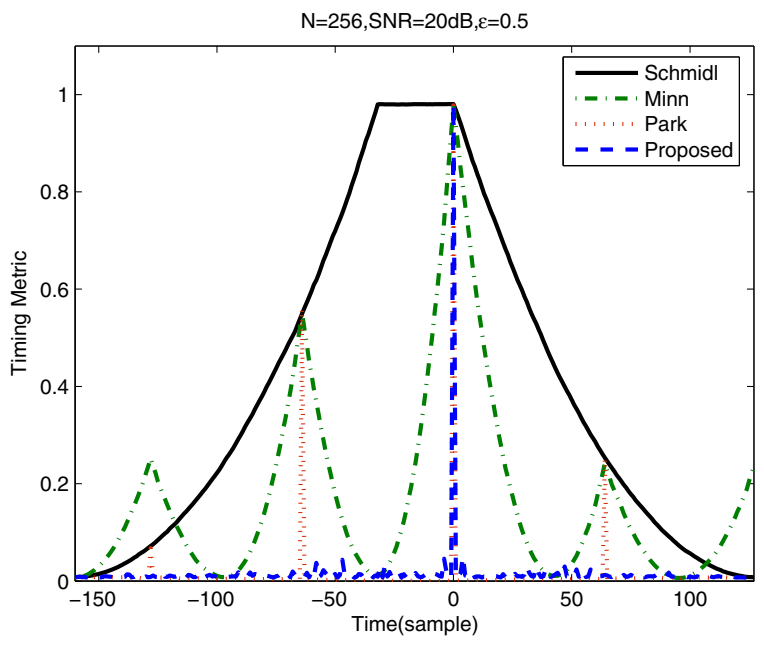

Fig. 2. Timing Metric of Proposed and Other Algorithm

At the transmitter, the new preamble can be defined as

$$
x_{k}^{\prime}=s_{k} \cdot x_{k}, \quad k=0,1, \ldots, N-1
$$

where $s_{i}$, drawn from PN sequence with a value either +1 or -1 , is the weighted factor of the $i$-th sample at IFFT outputs.

At the receiver, the received symbol is demodulated by the same PN sequence and the corresponding pairs of samples are correlated. The new timing metric is defined as:

$$
M(d)=\frac{|P(d)|^{2}}{|R(d)|^{2}}
$$

where

$$
\begin{gathered}
P(d)=\sum_{k=0}^{N / 2-1} s(k) s(k+N / 2) \cdot r^{*}(d+k) r(d+k+N / 2) \\
R(d)=\sum_{k=0}^{N / 2-1}|r(d+k+N / 2)|^{2}
\end{gathered}
$$

Since the PN sequence samples are generated randomly, the influence exerted by weighted factors would be removed only at the correct timing point, which is taken as the start of the useful part of the training symbol, and values around that particular point would be relatively small.

The timing metric of proposed method in AWGN environment is shown in Fig. 2. For comparison, Schmidl, Minn and Park timing estimators are also presented. The frequency offset is set to be 0.5 subcarrier spacing but it does not exert much influence on the timing estimators as they are robust to it. The correct timing point is indexed 0 in the figure. As expected, the proposed timing metric shaped like an impulse has neither a plateau nor side-peaks.

As soon as the correct timing point, i.e., $\theta$, is located, (12) could be simplified as follows:

$$
\begin{aligned}
& \Lambda_{2 K+1, \theta}= \\
& {\left[\left|\Phi_{\theta}(K \cdot \triangle f)\right|^{2} \cdots\left|\Phi_{\theta}(0)\right|^{2} \cdots\left|\Phi_{\theta}(-K \cdot \triangle f)\right|^{2}\right]^{\prime}}
\end{aligned}
$$




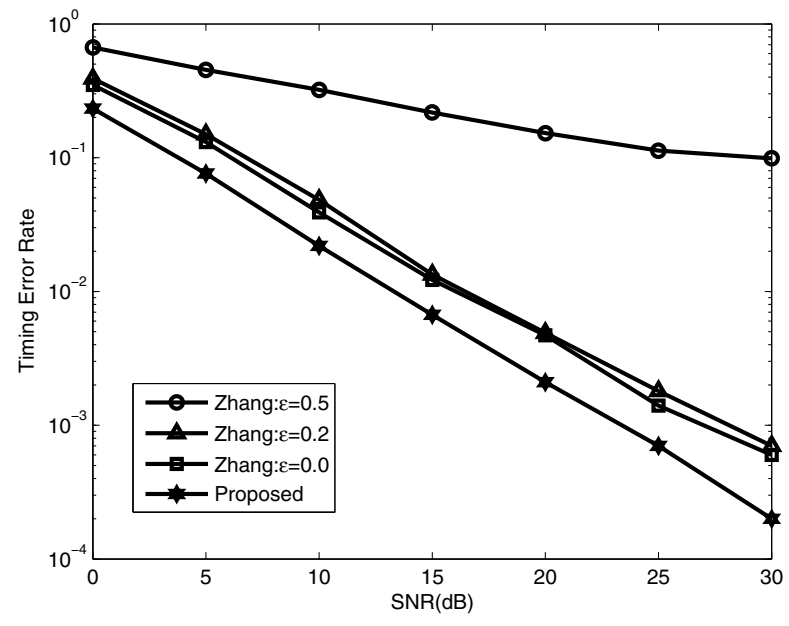

Fig. 3. TER of Zhang and Proposed Timing Estimator

where we get from (9) that

$$
\Phi_{\theta}(f)=\sum_{k=1}^{N / 2-1} e^{-j 2 \pi(f-\hat{f}) k}
$$

Then the frequency offset could be coarsely estimated. Stated in another way, to get $\hat{f}$, we now need to calculate (22), which is only one column of (12), and look for the maximum value.

Then in the tracking mode, the received samples are compensated with coarse frequency offset, and the fine adjustment algorithm (15) is taken to estimate the remaining frequency offset $\varepsilon_{R}$. The modification to the original timing estimator does not in the least influence the coarse and fine frequency offset estimation.

Overall, the proposed algorithm has some attractive features superior to Zhang's method.

1) The proposed timing estimator is robust to frequency offset, so it would not be affected by the value of $\triangle f$. What is more, The PN sequence weighted factors lead to an impulse shaped timing metric with neither plateau nor side-peaks.

2) The computational complexity is significantly reduced because the timing synchronization is performed in the beginning and the original complex matrix is simplified into one dimension. That is, only one column containing $(2 K+1)$ elements is needed in the acquisition mode, far smaller than that of Zhang's timing estimator, which is $(2 K+1) \cdot(L+1)$.

3) The algorithm could be further simplified if $K$ could be assigned a smaller value. This could be done by increasing $\triangle f$ slightly as long as the fine adjustment algorithm works. For instance, if $\triangle f$ is increased from 0.0025 to $0.003875, K$ would be $53.9 \%$ smaller, i.e., it is decreased from 200 to 130 .

Consequently, the proposed algorithm has the virtue of being accurate and affordable. The timing performance and

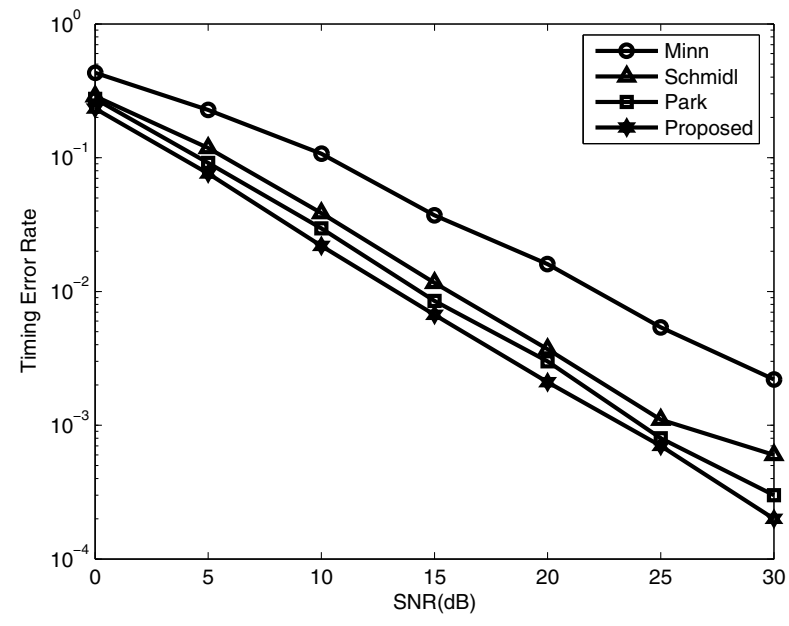

Fig. 4. TER of Proposed and Other Timing Estimator

computational complexity is further illustrated by computer simulations.

\section{Simulations And Analysis}

In this paper, a wireless system operating at $5 \mathrm{GHz}$ with bandwidth of $5 \mathrm{MHz}$ and Maximum Doppler Shift of 48.1 $\mathrm{Hz}$ is assumed for simulations. An outdoor dispersive, fading channel is modeled as 5 independent Rayleigh fading paths with path delay $\tau_{i}$ of $0,3,5,9$, and 12 samples and path gains given by $h_{i}=\exp \left(-\tau_{i}\right)$. The length of the preamble is 256 and that of CP is 32. QPSK modulation is employed and 10000 simulations are run.

\section{A. Timing Synchronization Analysis}

As we know, perfect timing point estimation is crucial for the operation of the fine adjustment algorithm. In Fig. 3, the performance of different timing estimators are evaluated in terms of timing error rate (TER). For Schmidl [2] and Minn [4] algorithm, TER is equal to the probability that the estimated starting point is outside CP. For Zhang [1], Park [5] and the proposed algorithm, TER is the probability that the estimated starting point is not the correct timing point $\theta$.

Fig. 3 indicates that Zhang's timing estimator is very sensitive to remaining frequency offset and has a large TER even though the frequency offset is perfectly estimated $\left(\varepsilon_{R}=0\right)$ in the acquisition stage. By comparison, the proposed algorithm is more accurate than Zhang's timing algorithm. When SNR is low, improvement about $3 \mathrm{~dB}$ and $4 \mathrm{~dB}$ of the proposed timing estimator compared to [1] could be obtained when the remaining carrier frequency offset $\varepsilon_{R}$ equals 0 and 0.2 , respectively. With SNR of $15 \mathrm{~dB}$, TER of proposed algorithm is 0.0067 , while TER of Zhang's method is $0.0122,0.0134$ and 0.2177 when $\rho$ equals $0,0.2$ and 0.5 , respectively. This is due to the improved timing estimator robust to $\varepsilon_{R}$ and free from side-peaks.

From Fig. 4 it is also clear that the proposed algorithm has better performance than Schmidl's, Minn's and Park's. When 
TABLE I

COMPUTATIONAL COMPLEXITy ANALYSIS

\begin{tabular}{|l|c|c|}
\hline & Zhang & Proposed \\
\hline comp mul & $(2 K+2)(N / 2-1)(L+1)$ & $(K+1)(N-2)+(L+1)(N+1.75)$ \\
\hline comp div & $(N / 2-1)(L+1)$ & $(N / 2+L)$ \\
\hline comp add & $(2 K+1)(N / 2-2)(L+1)$ & $(2 K+1)(N / 2-2)+(L+1)(N-2)$ \\
\hline exp oper & $(2 K+1)(N / 2-1)(L+1)$ & $(2 K+1)(N / 2-1)$ \\
\hline mod oper & $(N / 2+2 K)(L+1)$ & $(N / 2+2 K)$ \\
\hline
\end{tabular}

SNR is low, improvement about $1 \mathrm{~dB}$ and $2 \mathrm{~dB}$ of the proposed timing estimator compared to [5] and [2], respectively, could be obtained. When SNR is 15dB, TER of proposed, Schmmidl, Minn and Park algorithm is 0.0067, 0.01160 .0371 and 0.0085 .This improvement results from the following factors:

1) It does not have a plateau created in [2].

2) It does not have side-peaks inherent in [4] and [5].

3) The timing metric is shaped as an impulse, which is better than [4] even though side-peaks are not taken into account.

\section{B. Frequency Offset Estimation Analysis}

In the tracking mode, the fine adjustment algorithm is adopted to get an estimation of $\varepsilon_{R}$ more accurate than that of Schmidl. In the AWGN channel, performance improvement about $1 \mathrm{~dB}$ of the fine adjustment algorithm compared to Schmidl's algorithm is obtained when $\rho$ is 1 , while in multipath fading channel, the improvement would be up to $4.6 \mathrm{~dB}$ in a low SNR [1].

It is noteworthy that Zhang's frequency offset estimator depends on the detection of correct timing point, i.e., $\theta$. Therefore the improved timing synchronization algorithm makes Zhang's frequency offset estimation method much more feasible and reliable.

\section{Computational Complexity Analysis}

Detailed analysis on computational complexity listed in Table I illustrates the proposed algorithm's efficiency. The computational complexity of Zhang algorithm is significantly reduced by preceding the timing synchronization since it enables the original matrix to be simplified into one dimension. On one hand, it saves considerable time to perform the complex algorithm. On another hand, it reduces the overall system cost by decreasing the number of devices such as expensive memory elements. For example, when $N=$ $256, K=160, L+1=160, \triangle f=0.003125$, the number of elements in (12) and (22) is 51360 and 321, respectively, and the calculation amount of complex multiplication is $7866.3 \%$ less than that of the original algorithm. Note that in the table, comp mul, comp div, comp add, exp oper and mode oper is the abbreviation for complex multiplication, complex division, complex addition, exponential operation and modulo operation, respectively.

\section{CONCLUSION}

An efficient joint frame timing and frequency offset estimation algorithm for OFDM systems as a modification to Zhang's method has been proposed. By using PN sequence weighted factors, an impulse shaped timing metric robust to frequency offset and free from side-peaks is obtained. Performing timing synchronization first, we are able to simplify Zhang's algorithm significantly with better timing synchronization performance and a more reliable frequency offset estimator. Simulation results and complexity analysis have proved the accuracy and efficiency of the proposed algorithm.

\section{REFERENCES}

[1] Z. Zhang, K. Long, M. Zhao, and Y. Liu, "Joint frame synchronization and frequency offset estimation OFDM systems," IEEE Trans. Broadcast., vol. 51, pp. 139-143, Sep. 2005.

[2] T. M. Schmidl and D. C. Dox, "Robust frequency and timing synchronization for OFDM," IEEE Trans. Commun., vol. 45, pp. 1613-1621, Dec. 1997.

[3] Y. H. Kim, I. Song, S. Yoon, and S. R. Park, "An efficient frequency offset estimator for OFDM systems and its performance characteristics," IEEE Trans. Veh. Technol., vol. 50, pp. 1307-1312, Sep. 2001.

[4] H. Minn and V. K. Bhargava, "A simple and efficient timing offset estimation for OFDM systems," in Proc. IEEE Vehicular Conference (VTC' 2000), vol. 1, Tokyo, Japan, Jan. 2000, pp. 51-55.

[5] B. Park, H. Cheon, C. Kang, and D. Hong, "A novel timing estimation method for OFDM systems," IEEE Commun. Lett., vol. 7, pp. 239-241, May 2003.

[6] G. Ren, Y. Chang, H. Zhang, and H. Zhang, "Synchronization method based on a new constant envelope preamble for OFDM systems," IEEE Trans. Broadcast., vol. 51, pp. 139-143, Mar. 2005.

[7] A. Czylwik, "Synchronization for systems with antenna diversity," in Proc. IEEE Vehicular Conference (VTC' 1999), vol. 2, Amsterdam, Netherlands, Sep. 1999, pp. 728-732. 